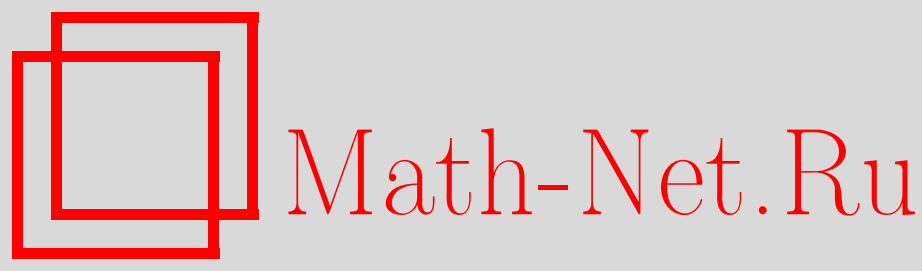

А. А. Андрианов, В. А. Андрианов, Д. Эсприу, Ш. Планельс, Аномальное усиление выхода дилептонов в центральных столкновениях тяжелых ионов вследствие локального нарушения четности, ТМФ, 2012, том 170, номер 1, 22-33

DOI: https://doi.org/10.4213/tmf6743

Использование Общероссийского математического портала Math-Net.Ru подразумевает, что вы прочитали и согласны с пользовательским соглашением http://www.mathnet.ru/rus/agreement

Параметры загрузки:

IP : 54.166 .219 .16

26 апреля 2023 г., $17: 10: 55$

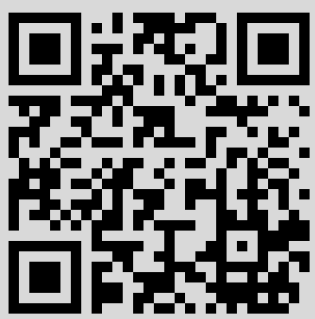



Д. Эсприу ${ }^{\dagger \dagger}$, Ш. Планельс ${ }^{\dagger}$

\title{
АНОМАЛЬНОЕ УСИЛЕНИЕ ВЫХОДА ДИЛЕПТОНОВ В ЦЕНТРАЛЬНЫХ СТОЛКНОВЕНИЯХ ТЯЖЕЛЫХ ИОНОВ ВСЛЕДСТВИЕ ЛОКАЛЬНОГО НАРУШЕНИЯ ЧЕТНОСТИ
}

\begin{abstract}
Предложено новое объяснение избытка дилептонов, наблюдаемого в плотной (горячей) ядерной материи в области инвариантных масс ниже 1 ГэВ. Приводятся доводы в пользу того, что наличие локального нарушения четности благодаря изосинглетному и/или изотриплетному псевдоскалярному конденсату, зависящему от времени, может существенно модифицировать дисперсию фотонов и векторных мезонов, распространяющихся в такой среде, в результате чего происходит аномально большой выход электрон-позитронных пар по сравнению с обычными теоретическими предсказаниями, основанными на "коктейле" адронных процессов. Предложены различные тесты, которые могут доказать или опровергнуть этот эффект.
\end{abstract}

Ключевые слова: сверхплотная барионная материя при больших температурах, спонтанное нарушение четности.

Для двоих из нас (А.А. Андрианова и В.А. Андрианова) настоящий интерес к изучению сложной природы сильных взаимодействий возник после изучения идеи цветных степеней свободы квантовой хромодинамики, предложенной в работе [1], в которую вклад А.Н. Тавхелидзе был существенным. Наши личные исследования начались, когда мы стали участвовать в международных семинарах "Кварки", которые были организованы А.Н. Тавхелидзе и его коллегами и учениками. Мы посвящаем нашу работу его памяти.

*Санкт-Петербургский государственный университет, Санкт-Петербург, Россия. E-mail: andrianov@icc.ub.edu,v.andriano@rambler.ru

${ }^{\dagger}$ Departament d'Estructura i Constituents de la Matèria and Institut de Ciències del Cosmos, Universitat de Barcelona Martíi Franquès 1, Barcelona, Spain.

E-mail: espriu@ecm.ub.es, xumeu@icc.ub.edu

${ }^{\ddagger}$ CERN, Geneva, Switzerland 


\section{1. ВВЕДЕНИЕ}

В последнее десятилетие в ряде экспериментов по столкновению тяжелых ионов был обнаружен аномальный выход лептонных пар с инвариантной массой $<1$ ГэВ в области малых быстрот и средних поперечных импульсов [2]-[5] (см. обзоры в работах [6], [7]). Этот эффект виден лишь в центральных или почти центральных столкновениях. Большинство исследований относится к парам $e^{+} e^{-}$, однако избыточный выход димюонных пар тоже был найден выше димюонного порога. Из сравнения протон-протонных и протон-ядерных столкновений было надежно установлено, что такое усиление выхода лептонных пар является, без сомнения, эффектом ядерной среды [6]. Для энергий, достижимых на установке GSI (несколько ГэВ на нуклон в экспериментах HADES [6]), эффект был интерпретирован как следствие усиленного выхода $\eta$-мезонов в протон-нейтронном рассеянии [5]. Для бо́льших энергий, достижимых на установках CERN SPS и BNL RHIC (порядка 100 ГэВ на нуклон в экспериментах CERES, HELIOS/3, NA60 [2], [3] и PHENIX [4]), аномальный выход дилептонов все еще не получил удовлетворительного объяснения в рамках известных механизмов в адронной феноменологии [6], [7].

Следуя [4], мы делим область инвариантных масс на высокоэнергетическую (выше 3.2 ГэВ), низкоэнергетическую (ниже 1.2 ГэВ) и промежуточную области. В низкоэнергетической области усиление могло бы быть объяснено возможной модификацией свойств мезонов в ядерной среде [8]-[11], а также частично протон-нейтронным рассеянием [5]. Наряду с этим распад $\rho$-мезона доминирует в окрестности мезонных полюсов $\rho-\omega$, однако эффекты среды уменьшения массы и/или уширения резонанса не могут объяснить впечатляющее усиление выхода дилептонов в данных PHENIX [4] на фактор 4-7 в зависимости от величины $p_{T}$ и центральности взаимодействия.

В настоящей работе мы обсуждаем совершенно другое объяснение этого усиления (предварительно очерченное в работе [12]). Мы предполагаем, что этот эффект может быть проявлением локального нарушения четности (ЛНЧ) в сталкивающихся ядрах в результате образования псевдоскалярного, изосинглетного или нейтрального изотриплетного, классического фонового поля, величина которого зависит от динамики столкновения. Идея пионной конденсации при конечной барионной плотности обсуждалась много лет назад Мигдалом и др. [13]. Недавно возможность образования изосинглетного псевдоскалярного конденсата при больших барионных плотностях была обоснована в работах [14]. Также в работах [15] было предложено считать, что изотриплетный псевдоскалярный фон мог бы появиться в результате флуктуаций топологического заряда большого масштаба, приводящих к так называемому киральному магнитному эффекту, исследованному в моделированиях КХД на решетке [16] и, по-видимому, обнаруженному в экспериментах STAR на RHIC [17] (отметим, однако, что этот вопрос еще далек от окончательного решения). Обсуждаемое усиление также может иметь отношение к псевдоскалярным доменным стенкам [18]. Однако тот факт, что наблюдаемый дилептонный выход отсутствует для периферийных столкновений (где киральный магнитный эффект должен быть более заметным) и максимален в центральных столкновениях, позволяет считать, что он может соответствовать образованию локально термодинамической фазы, в которой нарушена пространственная четность. 


\section{2. ЗАВИСЯЩЕЕ ОТ ВРЕМЕНИ ПСЕВДОСКАЛЯРНОЕ ПОЛЕ ИНИЦИИРУЕТ АНОМАЛЬНЫЙ ВЫХОД ДИЛЕПТОНОВ}

В работах [19] было показано, что псевдоскалярное поле, медленно развивающееся во времени, существенно меняет электромагнитные свойства вакуума. В частности, энергичный фотон, распространяющийся в этом фоновом поле, может распадаться на массовой поверхности в дилептоны. Этот же механизм, обобщенный на векторные мезоны, предлагается в настоящей статье для объяснения аномального выхода пар $e^{+} e^{-}$в низкоэнергетической области, т. е. при 0.15 ГэВ $<M_{e e}<1$ ГэВ, для центральностей $0-20 \%$ и для промежуточных значений $p_{T}<1$ ГэВ [4], для которых расхождение между теорией и экспериментом более загадочно.

Для области $M_{e e}>300$ МэВ мы включаем векторные мезоны $\rho_{0}$ и $\omega$ в $S U(2)$-флейворный сектор (мы не рассматриваем $\phi$-мезон, который менее значим для выбранной нами области $M_{e e}$ ). Соответствующей основой для описания электромагнитных взаимодействий адронов при низких энергиях является модель векторной доминантности (МВД) [9], [20]. Мы будем предполагать, что зависящее от времени, но приближенно пространственно однородное псевдоскалярное поле $a(t)$ индуцируется при плотностях, достижимых в столкновениях тяжелых ионов, и мы определим соответствующий ему 4-вектор $\hat{\zeta}_{\mu} \simeq \partial_{\mu} a \simeq \delta_{0 \mu} \hat{\zeta}$ для дальнейшего использования. Мы рассматриваем возможность того, что поле $a(t)$ является либо изосинглетом, либо изотриплетом, либо даже их смесью, однако детальные вычисления будут представлены только для случая изосинглетного фонового поля.

Соответствующий кинетический член для лагранжиана векторных полей $V_{\mu}(x)$ в псевдоскалярном зависящем от времени фоновом поле содержит максвелловский и массовые члены, дополненные членом взаимодействия Черна-Саймонса:

$$
\begin{gathered}
\mathcal{L}_{\text {int }}=\bar{q} \gamma_{\mu} V^{\mu} q, \quad V_{\mu} \equiv-e A_{\mu} Q+\frac{1}{2} g_{\omega} \omega_{\mu} \mathbf{I}+g_{\rho} \rho_{\mu} \frac{\tau_{3}}{2}, \\
\left(V_{\mu, a}\right) \equiv\left(A_{\mu}, \omega_{\mu}, \rho_{\mu} \equiv\left(\rho_{0}\right)_{\mu}\right),
\end{gathered}
$$

где $Q=\tau_{3} / 2+\mathbf{I} / 6, g_{\omega} \simeq g_{\rho} \equiv g \simeq 6$. Эти значения извлекаются из распадов векторных мезонов. Векторные поля нормируются на обычные кинетические члены в вакууме:

$$
\begin{aligned}
& \mathcal{L}_{\text {kin }}=-\frac{1}{4}\left(F_{\mu \nu} F^{\mu \nu}+\omega_{\mu \nu} \omega^{\mu \nu}+\rho_{\mu \nu} \rho^{\mu \nu}\right)+\frac{1}{2} V_{\mu} \widehat{m}^{2} V^{\mu}, \\
& m_{a, b}^{2}=m_{\mathrm{V}}^{2}\left(\begin{array}{ccc}
10 e^{2} / 9 g^{2} & -e / 3 g & -e / g \\
-e / 3 g & 1 & 0 \\
-e / g & 0 & 1
\end{array}\right), \quad \operatorname{det} \widehat{m}^{2}=0,
\end{aligned}
$$

где $m_{\mathrm{V}}^{2}=m_{\rho}^{2}=2 g_{\rho}^{2} f_{\pi}^{2} \simeq m_{\omega}^{2}$. Матрица масс отражает соотношения МВД на уровне кварковой модели [20], [9].

Пространственно нечетный вклад обеспечивается членом Черна-Саймонса

$$
\mathcal{L}_{\mathrm{CS}}(k)=-\frac{1}{4} \varepsilon^{\mu \nu \rho \sigma} \operatorname{tr} \hat{\zeta}_{\mu} V_{\nu}(x) V_{\rho \sigma}(x)=\frac{1}{2} \operatorname{tr} \hat{\zeta} \epsilon_{j k l} V_{j} \partial_{k} V_{l}=\frac{1}{2} \zeta \epsilon_{j k l} V_{j, a} N_{a b} \partial_{k} V_{l, b},
$$

который вызывает смешивание между фотонами и векторными мезонами, индуцированными ЛНЧ. Для изосинглетного псевдоскалярного фонового поля $\hat{\zeta}=9 \zeta \mathbf{I} / 5$, 
и матрица смешивания имеет вид

$$
N_{a b} \simeq\left(\begin{array}{ccc}
1 & -3 g / 10 e & -9 g / 10 e \\
-3 g / 10 e & 9 g^{2} / 10 e^{2} & 0 \\
-9 g / 10 e & 0 & 9 g^{2} / 10 e^{2}
\end{array}\right), \quad \operatorname{det} \widehat{N}=0
$$

Примечательно, что $\widehat{N} \sim \widehat{m}^{2}$. Простые рассмотрения по порядку величины указывают, что $\zeta \sim \alpha \tau^{-1} \sim 1$ МэВ, если взять $\tau=1 \Phi_{\mathrm{M}}$.

Для изотриплетного псевдоскалярного фонового поля $\hat{\zeta}=3 \zeta \tau_{3}$, а соответствующая матрица Черна-Саймонса принимает форму

$$
N_{a b}^{\pi} \simeq\left(\begin{array}{ccc}
1 & -3 g / 2 e & -g / 2 e \\
-3 g / 2 e & 0 & 3 g^{2} / 2 e^{2} \\
-g / 2 e & 3 g^{2} / 2 e^{2} & 0
\end{array}\right), \quad \operatorname{det} \widehat{N}^{\pi}=0 .
$$

Коэффициенты МВД в выражениях (3), (4) получены из аномального действия Весса-Зумино [21] и относятся к феноменологии радиационных распадов векторных мезонов [22]. Отношения матричных элементов для изотриплетного конденсата в (4) находятся в согласии с экспериментальными константами распада для процессов $\pi_{0} \rightarrow \gamma \gamma, \omega \rightarrow \pi_{0} \gamma, \rho_{0} \rightarrow \pi_{0} \gamma$ [21] и для распада $\omega \rightarrow \pi \pi \pi$ [23], взятыми из [24]. Аналогично элементы в (3) могут быть в принципе оценены из распадов $\eta \rightarrow \gamma \gamma, \eta^{\prime} \rightarrow \gamma \gamma, \omega \rightarrow \eta \gamma, \rho_{0} \rightarrow \eta \gamma$. Однако феноменологически существует эффект сильного смешивания $\eta_{8}-\eta_{0}$, который должен быть в конце концов разрешен в схеме $S U(3)$-флейворов [25]. Лишь отношение ширин рападов $\omega \rightarrow \eta \gamma, \rho_{0} \rightarrow \eta \gamma$ мало чувствительно к смешиванию и подтверждает недиагональные элементы (3). В настоящей работе мы пренебрегаем этим смешиванием и ограничиваемся случаем $S U(2)$. Мы отдаем себе отчет в том, что лагранжиан векторных мезонов в горячей и плотной материи может слегка отличаться от вакуумной формы, принятой в данном случае, однако мы надеемся, что отклонения можно ожидать исключительно в массах и ширинах распада мезонов, а не в аномальных константах связи в лагранжиане Becса-Зумино, так как эти вершины взаимодействия обычно являются устойчивыми по отношению к радиационным эффектам. Кроме того, мы используем здесь оценки в пределе большого числа “цветов" $N_{\mathrm{c}}$, которые оправдывают пренебрежение возможной зависимостью констант от химического потенциала и температуры.

Более того, как ранее упоминалось, мы ограничиваемся случаем изосинглетного псевдоскалярного фонового поля $a(t)$. В этом случае можно найти уравнения на массовой поверхности для векторных мезонов

$$
\begin{gathered}
K_{a b}^{\mu \nu} V_{\nu, b}=0, \quad k^{\nu} V_{\nu, b}=0, \\
K^{\mu \nu} \equiv g^{\mu \nu}\left(k^{2} \mathbf{I}-\widehat{m}^{2}\right)-k^{\mu} k^{\nu} \mathbf{I}-i \varepsilon^{\mu \nu \rho \sigma} \zeta_{\rho} k^{\sigma} \widehat{N},
\end{gathered}
$$

отбирающие три физические поляризации для массивных векторных мезонов. Фактически эти три поляризации дают вклад в пропагаторы векторных полей, поскольку они связаны с сохраняющимися фермионными токами. Продольная поляризация $\varepsilon_{\mathrm{L}}^{\mu}$ ортогональна к $k_{\mu}$ и к члену Черна-Саймонса:

$$
\varepsilon_{\mathrm{L}}^{\mu}=\frac{\zeta^{\mu} k^{2}-k^{\mu}(\zeta \cdot k)}{\sqrt{k^{2}\left((\zeta \cdot k)^{2}-\zeta^{2} k^{2}\right)}}, \quad \varepsilon_{\mathrm{L}} \cdot \varepsilon_{\mathrm{L}}=-1,
$$


для $k^{2}>0$. Масса этого состояния остается неискаженной. С другой стороны, поперечные (круговые) поляризации $\varepsilon_{ \pm}^{\mu}$ удовлетворяют соотношению

$$
K_{\nu}^{\mu} \varepsilon_{ \pm}^{\nu}=\left(k^{2} \mathbf{I}-\widehat{m}^{2} \pm \sqrt{(\zeta \cdot k)^{2}-\zeta^{2} k^{2}} \widehat{N}\right) \varepsilon_{ \pm}^{\mu}
$$

Спектр может быть найден после одновременной диагонализации матриц $\widehat{m}^{2}, \widehat{N}$ после ограничения на случай $\zeta_{\mu} \simeq(\zeta, 0,0,0)$ :

$$
\widehat{N}=\operatorname{diag}\left[0, \frac{9 g^{2}}{10 e^{2}}, \frac{9 g^{2}}{10 e^{2}}+1\right], \quad \widehat{m}^{2}=m_{\mathrm{V}}^{2} \operatorname{diag}\left[0,1,1+\frac{10 e^{2}}{9 g^{2}}\right],
$$

а именно

$$
k_{0}^{2}-\vec{k}^{2}=m_{\mathrm{V}}^{2} \pm \frac{9 g^{2}}{10 e^{2}} \zeta|\vec{k}| \simeq m_{\mathrm{V}}^{2} \pm 360 \zeta|\vec{k}| \equiv m_{\mathrm{V}, \pm}^{2}
$$

Таким образом, в случае изосинглетного псевдоскалярного фонового поля безмассовые фотоны не искажаются, когда смешиваются с массивными векторными мезонами. В свою очередь, массивные векторные мезоны расщепляются на три поляризации с массами $m_{\mathrm{V},-}^{2}<m_{\mathrm{V}, \mathrm{L}}^{2}<m_{\mathrm{V},+}^{2}$. Это расщепление недвусмысленно указывает на ЛНЧ, также как и на нарушение лоренц-инвариантности, из-за зависимости фонового поля от времени. Для достаточно больших $|\vec{k}| \geqslant 10 e^{2} m_{\mathrm{V}}^{2} / 9 g^{2} \zeta \simeq m_{\mathrm{V}}^{2} / 360 \zeta$ состояния векторных мезонов с отрицательной поляризацией становятся тахионами. Однако их групповая скорость остается меньше, чем скорость света [26], при условии, что $\zeta<20 e^{2} m_{\mathrm{V}} / 9 g^{2} \simeq m_{\mathrm{V}} / 180 \approx 4.3$ МэВ. Для бо́льших значений $\zeta$ вакуумное состояние оказывается нестабильным, а именно: эффекты поляризации дают мнимый вклад в вакуумную энергию.

Отметим, что положения резонансных полюсов для положительно и отрицательно поляризованных мезонов оказываются движущимися с волновым вектором $|\vec{k}|$, и поэтому они обнаруживают себя как уширенные резонансы. Увеличение резонансной области потенциально приводит к существенному усилению их вклада в выход дилептонов в стороне от положения их номинального вакуумного резонанса.

Разъясним анзац постоянного фонового поля Черна-Саймонса для описания явления ЛНЧ. Можно ожидать следующую временну́ю зависимость псевдоскалярного конденсата в столкновениях тяжелых ионов. На первой стадии столкновений ядерная материя является сжатой и нагретой (в течение времени $\tau_{\mathrm{h}}$ ), появляется конденсат и он предположительно растущий. Можно экстраполировать его поле линейной функцией с наклоном $\sim \zeta_{\mathrm{h}}$. На следующем этапе, в пике плотности энергии, псевдоскалярное фоновое поле является почти постоянным, т. е. не дает вклада в ЛНЧ. В конце концов ядерная материя (файербол) охлаждается (в течение времени $\tau_{\mathrm{c}}$ ), конденсат уменьшается и исчезает на стадии вымораживания. Мы снова экстраполируем его линейной функцией, но с другим наклоном $\sim \zeta_{\text {c }}$ (противоположного знака). Таким образом, параметр $\zeta$ меняет знак, однако для самого эффекта ЛНЧ только его модуль оказывается важным из-за того, что изменение его знака вызывает смену поляризаций, но не влияет на расщепление масс для разных поляризаций. Соответственно мы можем использовать среднее $|\zeta| \simeq\left(\left|\zeta_{\mathrm{h}}\right| \tau_{\mathrm{h}}+\left|\zeta_{\mathrm{c}}\right| \tau_{\mathrm{c}}\right) /\left(\tau_{\mathrm{h}}+\tau_{\mathrm{c}}\right)$ для сжатой и охлажденной фаз, что мы на самом деле и делаем. Конечно, более точное рассмотрение должно быть основано на вычислениях формирования и эволюции файербола, мы планируем сделать это в последующей работе. 


\section{3. УСИЛЕНИЕ ВЫХОДА ДИЛЕПТОНОВ ДЛЯ ПОПЕРЕЧНЫХ ПОЛЯРИЗАЦИЙ ВЕКТОРНЫХ МЕЗОНОВ В СИЛУ НАРУШЕНИЯ Р-ЧЕТНОСТИ В СРЕДЕ}

Выход пар $e^{+} e^{-}$, обусловленный $\rho$ - и $\omega$-мезонами, имеет ту же форму, как и в работах [9], но с модифицированными пропагаторами в силу ЛНЧ, в соответствии с нашим предыдущим обсуждением:

$$
\begin{aligned}
\frac{d N_{e e}}{d^{4} x d M} \simeq \sum_{\mathrm{V}} & c_{\mathrm{V}} \frac{\alpha^{2} \Gamma_{\mathrm{V}} m_{\mathrm{V}}^{2}}{3 \pi^{2} g^{2} M^{2}}\left(\frac{M^{2}-n_{\mathrm{V}}^{2} m_{\pi}^{2}}{m_{\mathrm{V}}^{2}-n_{\mathrm{V}}^{2} m_{\pi}^{2}}\right)^{3 / 2} \times \\
& \times \sum_{\epsilon} \int_{M}^{\infty} d k_{0} \frac{\sqrt{k_{0}^{2}-M^{2}}}{e^{k_{0} / T}-1} \frac{m_{\mathrm{V}, \epsilon}^{4}\left(1+\Gamma_{\mathrm{V}}^{2} / m_{\mathrm{V}}^{2}\right)}{\left(M^{2}-m_{\mathrm{V}, \epsilon}^{2}\right)^{2}+m_{\mathrm{V}, \epsilon}^{4} \Gamma_{\mathrm{V}}^{2} / m_{\mathrm{V}}^{2}}
\end{aligned}
$$

где $n_{\mathrm{V}}=2,3$ для $\rho$ - и $\omega$-мезонов соответственно. Для выхода дилептонов в $\omega$-мезонном канале это выражение используется с трехпионным разрезом со своей собственной константой $c_{\omega}$ и кинематическими коэффициентами, характерными для основного распада $\omega$.

Коэффициенты $c_{\rho}, c_{\omega}$ параметризуют феноменологическим образом полные сечения для рождения векторных мезонов. Поскольку они не известны с достаточной точностью, в частности не известны их значения вне массовой поверхности, относительные веса используются как свободные параметры в адронном "коктейле" [2], [3], [27], [28]. Эта процедура используется в экспериментах NA60 и PHENIX, и здесь мы будем ей также следовать. Обычный "коктейль" содержит веса, нормируемые на результат периферийных столкновений (приблизительно согласованно с существующими нуклонными данными по $p p$ - и $p$-ядерным столкновениям). Для полуцентральных и центральных столкновений, в частности при малых $p_{T}$, коэффициент $\rho / \omega$ следует увеличить, по крайней мере, на фактор 1.6 в случае эксперимента NA60 [2] или приблизительно на 1.8 в эксперименте PHENIX [4], [29].

Простое тепловое усреднение по энергиям пионного газа [30] (предположительно представляющее файербол после столкновения тяжелых ионов) также было включено. Коэффициент $c_{\mathrm{V}}$ содержит некоторые комбинаторные факторы, разные для $\rho$ - и $\omega$-мезонов, а также долю выхода на единицу объема тепловых пионов, отвечающих за рождение векторных мезонов; кроме того, он зависит от химического потенциала [9].

Усиление выхода дилептонов вследствие ЛНЧ по сравнению с оценками обычного "коктейля" показано на рис. 1 для $T=150$ МэВ. В области 300 МэВ $<M<$ 900 МэВ виртуальные $\rho$-мезоны дают существенный вклад в получение пар $e^{+} e^{-}$из $\pi \pi$-слияния. Распады $\omega$-мезонов также важны, но они дают менее значительный вклад по сравнению с распадами $\rho$-мезонов, за исключением самой окрестности резонансного пика $\omega$. Кроме всего прочего, существует вклад и от распада Далица $\eta$-мезона, к которому мы вернемся позднее.

$\rho$-Мезон имеет сильную связь с $\pi \pi$-каналом, и его средняя длина пробега (1.3 Фм) существенно меньше, чем ожидаемый размер файербола адронного газа (5-10 $\left.\Phi_{\mathrm{M}}\right)$. Таким образом, эффекты среды для $\rho$-мезона должны быть наиболее важными для обеспечения избыточного выхода дилептонов. 


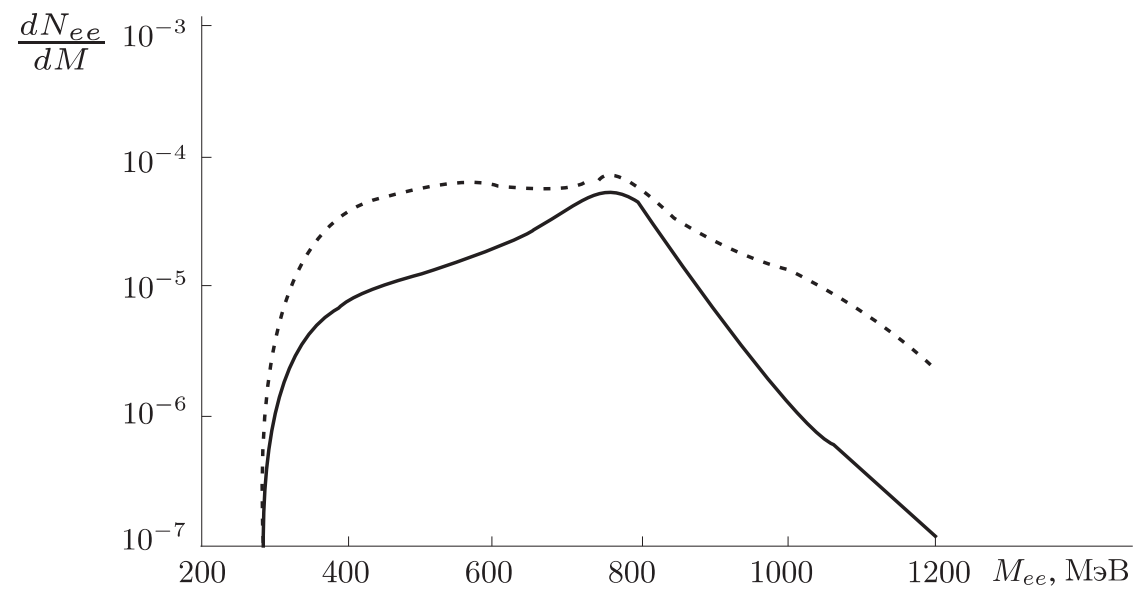

Рис. 1. Вклад $\rho$-мезонов в дилептонное рождение для случая пространственно четной ядерной материи ( $\zeta=0$; сплошная кривая) и для ЛНЧ ( $\zeta=2 \mathrm{MэВ;}$ штриховая кривая). Масштаб вертикальной оси нормирован на данные эксперимента PHENIX [4].

Заметим, что длина свободного пробега $\rho$-мезона (1.3 $\left.\Phi_{\mathrm{M}}\right)$ много короче, чем ожидаемый размер адронного файербола $\left(L=5-10 \Phi_{\mathrm{M}}\right)$. Время жизни файербола сравнимо: $\tau_{\mathrm{fb}} \simeq L$. В свою очередь, длина свободного пробега $\omega$-мезона в 17 раз больше, поэтому вероятность его распада в файерболе не слишком высока и увеличение выхода дилептонов в среде благодаря эффекту ЛНЧ относительно меньше.

Действительно, время, затраченное на рождение ультрарелятивистского резонанса шириной Г внутри файербола, соотносится с его длиной свободного пробега 1/Г в такой среде. Поэтому при $\Gamma \tau_{\mathrm{fb}} \ll 1$ возникает значительное подавление резонансного усиления $1 / \Gamma^{2}$. Около искаженного резонансного пика грубая оценка этого подавления дает $\sim \tau_{\mathrm{fb}}^{2} \Gamma^{2}$. Это подавление оказывает влияние на все долгоживущие частицы, такие как частицы, входящие в процессы Далица. Это представляет окончательную причину, по которой нет необходимости включать векторный резонанс $\phi$ в обсуждение. На рис. 2 представлено усиление дилептонного выхода в случае пренебрежения этой редукцией.

Тем не менее резонансный пик в основном насыщается распадами $\omega$-мезона и чистый эффект изображен на рис. 3. Сравнение с результатами эксперимента PHENIX указывает на то, что значение $\zeta$ в диапазоне 1-2 МэВ дает усиление в этой области, достаточное, чтобы объяснить избыток дилептонов.

\section{4. ПРОЦЕССЫ ДАЛИЦА}

При малых инвариантных массах $\left(<300 \mathrm{MэB)} \mathrm{процессы} \mathrm{Далица} \pi_{0} \rightarrow \gamma e^{+} e^{-}\right.$, $\eta, \eta^{\prime} \rightarrow \gamma e^{+} e^{-}$или $\eta, \eta^{\prime} \rightarrow \gamma \mu^{+} \mu^{-}$почти насыщают выход пар $l^{+} l^{-}$. Процесс Далица $\omega \rightarrow \pi l^{+} l^{-}$также важен. Как и раньше, для изосинглетного фонового поля только спектральные плотности виртуальных $\rho$ - и $\omega$-состояний модифицируются 


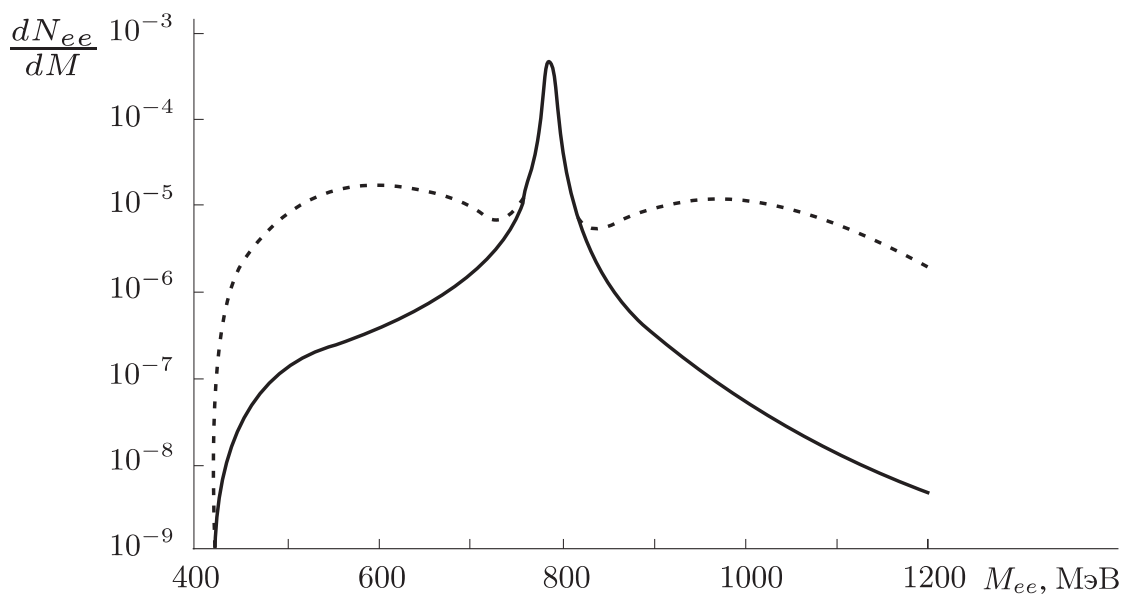

Рис. 2. Вклад $\omega$-мезона в выход дилептонов для пространственно четной ядерной материи ( $\zeta=0$; сплошная кривая) и для ЛНЧ ( $\zeta=2$ МэВ; штриховая кривая). Рассмотрен предел очень большого файербола.

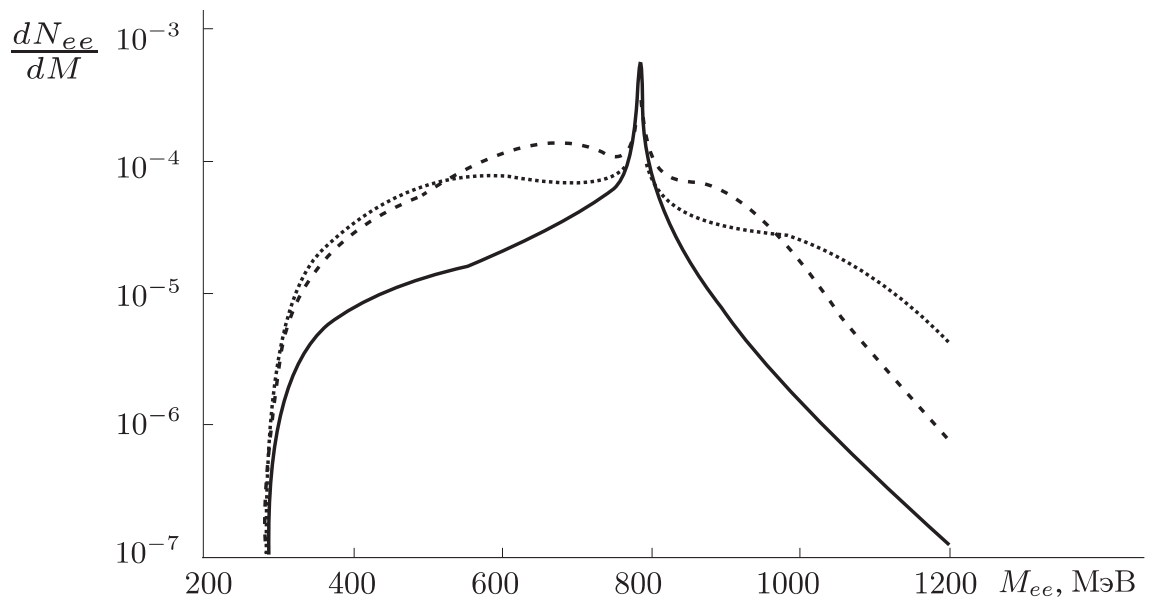

Рис. 3. Чистый вклад мезонов в выход дилептонов для пространственно четной ядерной материи ( $\zeta=0$; сплошная кривая) и для ЛНЧ ( $\zeta=1 \mathrm{MэВ,}$ штриховая кривая; $\zeta=2$ МэВ, пунктирная кривая). Нормировка пика $\rho+\omega$ выбрана по данным эксперимента PHENIX. 
псевдоскалярным фоновым полем в результате ЛНЧ. В этом случае аналог формулы Кролла-Вады [31] описывает парциальные ширины распада для поляризаций $L, \pm \rightarrow \epsilon=0, \pm 1$ искаженных векторных состояний:

$$
\begin{aligned}
& \frac{d N_{e e}}{d M d^{4} x d^{3} q} \simeq \sum_{\mathrm{V}, P} c_{P} \frac{\alpha^{3} m_{P}^{3}}{144 \pi^{4} f_{P}^{2} M}\left(1-\frac{M^{2}}{m_{P}^{2}}\right)^{3} \sum_{\epsilon}\left|F\left(M, m_{\mathrm{V}, \epsilon}\right)\right|^{2} \equiv \frac{d \Gamma_{\gamma e e}}{d M} \\
& F\left(M, m_{\mathrm{V}, \epsilon}\right) \equiv\left[\left(1-\frac{M^{2}}{m_{\mathrm{V}, \epsilon}^{2}}\right)^{2}-i \frac{\Gamma_{\mathrm{V} e e}}{m_{\mathrm{V}}}\right]^{-1},
\end{aligned}
$$

где для массивных векторных мезонов (9)

$$
m_{\mathrm{V}, \epsilon}^{2} \simeq M_{\rho}^{2}+\epsilon 360 \zeta|\vec{k}|, \quad m_{\mathrm{V}}^{2} \equiv m_{\mathrm{V}, 0}^{2} \simeq M_{\rho}^{2}
$$

и $\Gamma_{\rho е e} / m_{\rho} \simeq 10^{-5}, \Gamma_{\omega e e} / m_{\omega} \simeq 0.8 \cdot 10^{-6}$. Коэффициенты $c_{P}$ корректируют отношение $c_{\eta}: c_{\pi} \simeq 4.3$ двухфотонных распадов $\pi, \eta[31]$, а также включают выход в единице объема псевдоскалярных мезонов [4]. Этот выход также должен быть усреднен по тепловому распределению псевдоскалярных мезонов. Положения резонансов $m_{\mathrm{V}, \pm}^{2}$ сдвигаются с волновым вектором $\vec{k}$, и поэтому конволюция с тепловым распределением делает их шире. В формуле (12) усиление вычисляется для очень больших файерболов ядерной материи и оно определенно переоценено. Поскольку время жизни мезонных резонансов существенно больше, чем время столкновения $\tau \sim 5-10$ Фм, следует ожидать сильное подавление вкладов нарушения четности за счет конечности объема. Однако можно ожидать видимое усиление $\sim \tau^{2} m_{\mathrm{V}, \epsilon}^{2}>1$ для $m_{\mathrm{V}, \epsilon}>100$ МэВ. Эта оценка справедлива для $\Gamma_{\mathrm{Vee}} \tau \ll 1$. Дополнительная редукция вызывается конволюцией с тепловым распределением.

Когда $\hat{\zeta} \neq 0$ и его изоспиновое содержание фонового поля $\hat{\zeta}$ произвольно, $\hat{\zeta}=$ $A \mathbf{I}+B \tau_{3}$, фотоны с положительной поляризацией ведут себя как узкие резонансы [19] с шириной $\Gamma_{\gamma} \simeq \alpha \zeta / 3$ и распадаются в пару $l^{+} l^{-}$выше порога распада при $|\vec{k}|>4 m_{l}^{2} / \zeta$. Этот механизм усиления мог бы доминировать для выхода пар $e^{+} e^{-}$ при относительно малых инвариантных массах (<300 МэВ), когда процессы Далица $\pi_{0} \rightarrow \gamma e^{+} e^{-}, \eta \rightarrow \gamma e^{+} e^{-}$насыщают выход $e^{+} e^{-}$. Однако для димюонов порог распада слишком высок, чтобы фотонные распады играли какую-либо роль в рождении димюонов.

\section{5. ВЫВОДЫ И ПЕРСПЕКТИВЫ ОТКРЫТИЯ ЛНЧ}

В заключение мы хотели бы подчеркнуть черезвычайную простоту подхода, представленного в работе. Предложенные подгонки основаны на значениях (эффективных температурах, нормировках и пр.), на которые ссылаются эксперименты. Имеется только один свободный параметр $\zeta$, который, как ожидается, зависит от характеристик столкновения. Следует также оговорить, что наличие ЛНЧ не исключает других поправок как за счет многочастичных взаимодействий, так и в результате влияния среды [9], не нарушающего $P$-четность. 
Суммируем, наконец, признаки и выделим возможные пути поиска ЛНЧ.

1. Поляризация. Выходы дилептонов для значений нивариантных масс выше и ниже полюса $\rho+\omega$ имеют преимущественно противоположные круговые поляризации. Таким образом, можно было бы искать асимметрии продольно и поперечно поляризованных состояний для различных $M$ в измерениях от события к событию. Эти измерения могут недвусмысленно обнаружить существование ЛНЧ.

2. Искаженные фотоны. При низких энергиях доминируют амплитуды массивных векторных мезонов, однако фотоны также должны обнаружить искажение, порожденное ЛНЧ. Фотоны с положительной поляризацией показывают разные импульсные пороги $\sim 4 m_{l}^{2} / \zeta$ обнаружения резонансного поведения для различных дилептонных типов [19]. Заметим, что подавление выхода дилептонов за счет конечного размера файербола является важным в случае фотонов.

3. Природа конденсата. Процесс смешивания фотонов с векторными мезонами является чувствительным к изоспину псевдоскалярного конденсата, и поэтому знание доли распадов искаженных фотонов помогает определить его изоспиновое содержание.

Таким образом, в псевдоскалярном фоновом поле, зависящем от времени, безмассовые фотоны с положительной поляризацией и массивные векторные мезоны ведут себя как уширенные резонансы после усреднения по тепловому распределению.

Наконец, заметим, что для изосинглетного псевдоскалярного фонового поля, зависящего от времени, пропагаторы векторных $\rho$ - и $\omega$-мезонов искажаются. Мы вычислили этот эффект и нашли, что он естественным образом приводит к избыточному выходу дилептонных пар в резонансной области $\rho+\omega$. Модифицированная спектральная функция $\rho$-мезона указывает на ее особенности, очень схожие с измеренными в экспериментах NA60 по выходу димюонов. Данные эксперимента PHENIX намного лучше описываются этим механизмом, чем подгонкой стандартного "коктейля" в резонансной области $\rho+\omega$. При очень малых инвариантных массах процессы Далица $\pi^{0} \rightarrow \gamma e^{+} e^{-}, \eta \rightarrow \gamma l^{+} l^{-}$и $\omega \rightarrow l^{+} l^{-} \pi^{0}$ насыщают адронный вклад в выход $l^{+} l^{-}$и усиливаются благодаря модификации векторных мезонных пропагаторов, индуцируемой ЛНЧ (мы предполагаем детально рассмотреть эти процессы в последующих публикациях). Только один свободный параметр $\zeta$ характеризует временну́ю эволюцию псевдоскалярного конденсата. Хорошая подгонка к экспериментальным значениям может быть получена для естественных значений параметра $\zeta$. Мы обсудили возможность того, что конденсат ЛНЧ является изотриплетным или смесью изотриплета и изосинглета, и предложили экспериментальные тесты для выявления ЛНЧ в столкновениях тяжелых ионов. Таким образом, оказывается, что механизм ЛНЧ подходит для естественного объяснения дилептонной аномалии в экспериментах PHENIX, CERES, NA60, а поиск его проявления в выходе дилептонов представляет собой интересный экспериментальный вызов и может дать более глубокое понимание свойств КХД в экстремальных условиях.

Благодарности. Работа выполнена при финансовой поддержке по проектам FPA2010-20807, 2009SGR502 и Consolider CPAN CSD2007-00042. А. А. Андрианов и В. А. Андрианов поддержаны также РФФИ (гранты № 09-02-00073_a, 10-02-00881_а), а также грантом СПбГУ 11.0.64.2010. 


\section{Список литературы}

[1] Н. Н. Боголюбов, Б. В. Струминский, А. Н. Тавхелидзе, О составных моделях в теории элементарных частии, Препринт D-1968, ОИЯИ, Дубна, 1965.

[2] P. Wurn et al. (CERES Collab.), Nucl. Phys. A, 590:1-2 (1995), 103-116; G. Agakichiev et al. (CERES/NA45 Collab.), Eur. Phys. J. C, 4:2 (1998), 231-247; R. Arnaldi et al. (NA60 Collab.), Phys. Rev. Lett., 96:16 (2006), 162302, 5 pp.

[3] R. Arnaldi et al. (NA60 Collab.), Phys. Rev. Lett., 96:16 (2006), 162302, 5 pp.

[4] A. Adare et al. (PHENIX Collab.), Phys. Rev. C, 81:3 (2010), 034911, 56 pp., arXiv: 0912.0244 .

[5] G. Agakichiev et al. (HADES Collab.), Phys. Rev. Lett., 98:5 (2007), 052302, 5 pp., arXiv: nucl-ex/0608031; Phys. Lett. B, 663:1-2 (2008), 43-48.

[6] K. O. Lapidus, V. M. Emel'yanov, Phys. Part. Nucl., 40:1 (2009), 29-48.

[7] I. Tserruya, Electromagnetic Probes, arXiv: 0903.0415.

[8] G. E. Brown, M. Rho, Phys. Rev. Lett., 66:21 (1991), 2720-2723.

[9] R. Rapp, J. Wambach, Chiral Symmetry Restoration and Dileptons in Relativistic Heavy-Ion Collisions, Advances in Nuclear Physics, 25, Kluwer, New York, 2000; W. Liu, R. Rapp, Nucl. Phys. A, 796:1-4 (2007), 101-121, arXiv: nucl-th/0604031; H. van Hees, R. Rapp, Nucl. Phys. A, 806:1-4 (2008), 339-387.

[10] W. Cassing, E. Bratkovskaya, Nucl. Phys. A, 807:3-4 (2008), 214-250, arXiv: 0712.0635; E. L. Bratkovskaya, W. Cassing, O. Linnyk, Phys. Lett. B, 670:4-5 (2009), 428-433, arXiv: 0805.3177.

[11] K. Dusling, I. Zahed, Nucl. Phys. A, 825:3-4 (2009), 212-221, arXiv: 0712.1982.

[12] A. A. Andrianov, V. Andrianov, D. Espriu, X. Planells, Abnormal dilepton yield from local parity breaking in heavy-ion collisions, arXiv: 1010.4688.

[13] А.Б. Мигдал, ЖЖЭТФ, 61:6 (1971), 2209-2224; 63:6 (1972), 1993-1999; R. F. Sawyer, Phys. Rev. Lett., 29:6 (1972), 382-385; D. J. Scalapino, Phys. Rev. Lett., 29:6 (1972), 386-388; G. Baym, Phys. Rev. Lett., 30:26 (1973), 1340-1342; А. Б. Мигдал, О. А. Маркин, И. И. Мишутин, ЖЭТФ, 66:2 (1974), 443-463.

[14] A. A. Andrianov, D. Espriu, Phys. Lett. B, 663:5 (2008), 450-455, arXiv: 0709.0049; A. A. Andrianov, V. A. Andrianov, D. Espriu, Phys. Lett. B, 678:4 (2009), 416-421, arXiv: 0904.0413.

[15] D. Kharzeev, R. D. Pisarski, M. H. G. Tytgat, Phys. Rev. Lett., 81:3 (1998), 512-515, arXiv: hep-ph/9804221; K. Buckley, T. Fugleberg, A. Zhitnitsky, Phys. Rev. Lett., 84:21 (2000), 4814-4817, arXiv: hep-ph/9910229; D. Kharzeev, Phys. Lett. B, 633:2-3 (2006), 260-264, arXiv: hep-ph/0406125; D. E. Kharzeev, L. D. McLerran, H. J. Warringa, Nucl. Phys. A, 803:3-4 (2008), 227-253, arXiv: 0711.0950.

[16] P. Buividovich, M. Chernodub, E. Luschevskaya, M. Polikarpov, Phys. Rev. D, 80:5 (2009), 054503, 14 pp., arXiv: 0907.0494.

[17] B. I. Abelev et al. (STAR Collab.), Phys. Rev. Lett., 103:25 (2009), 251601, 7 pp., arXiv: 0909.1739; S. A. Voloshin, J. Phys. Conf. Ser., 230:1 (2010), 012021, 8 pp., arXiv: 1003.1127.

[18] A. Gorsky, M. B. Voloshin, Remarks on decay of defects with internal degrees of freedom, arXiv: 1006.5423.

[19] A. A. Andrianov, D. Espriu, P. Giacconi, R. Soldati, JHEP, 09 (2009), 057, 14 pp., arXiv: 0907.3709; A. A. Andrianov, D. Espriu, F. Mescia, A. Renau, Phys. Lett. B, 684:2-3 (2010), 101-105, arXiv: 0912.3151.

[20] J. J. Sakurai, Ann. Phys., 11:1 (1960), 1-48; Currents and Mesons, Univ. Chicago Press, Chicago, 1969.

[21] N. Kaiser, U.-G. Meissner, Nucl. Phys. A, 519:4 (1990), 671-696; E. Truhlik, J. Smejkal, F. C. Khanna, Nucl. Phys. A, 689:3-4 (2001), 741-752, arXiv: nucl-th/0010080. 
[22] O. Dumbrajs, R. Koch, H. Pilkuhn, G. C. Oades, H. Behrens, J. J. de Swart, P. Kroll, Nucl. Phys. B, 216:2 (1983), 277-335.

[23] F. Klingl, N. Kaiser, W. Weise, Z. Phys. A, 356:1 (1996), 193-206, arXiv: hep-ph/9607431.

[24] K. Nakamura et al. (Particle Data Group), J. Phys. G, 37:7A (2010), 075021, 1422 pp.

[25] T. Feldmann, P. Kroll, B. Stech, Phys. Rev. D, 58:11 (1998), 114006, 8 pp., arXiv: hep-ph/9802409; Y.N. Klopot, A. G. Oganesian, O. V. Teryaev, Dispersive approach to Abelian axial anomaly, mixing of pseudoscalar mesons and symmetries, arXiv: 0911.0180.

[26] J. Alfaro, A. A. Andrianov, M. Cambiaso, P. Giacconi, R. Soldati, Internat. J. Mod. Phys. A, 25:16 (2010), 3271-3306, arXiv: 0904.3557.

[27] R. Arnaldi et al. (NA60 Collab.), Phys. Lett. B, 677:5 (2009), 260-266, arXiv: 0902.2547.

[28] R. Arnaldi et al. (NA60 Collab.), Eur. Phys. J. C, 61:4 (2009), 711-720, arXiv: 0812.3053.

[29] В.Л. Рыков, частное сообщение, 2010.

[30] E. L. Feinberg, Il Nuovo Cimento B, 34:2 (1976), 391-410; L. McLerran, T. Toimela, Phys. Rev. D, 31:3 (1985), 545-563.

[31] L. G. Landsberg, Phys. Rept., 128:6 (1985), 301-376. 